\title{
Recommandations d'antibiotiques par les médecins de cabinets médicaux, 2007-2011
}

\author{
Finley $\mathbf{R}^{\mathbf{1}^{*}}$ \\ ${ }^{1}$ Centre des maladies infectieuses d'origine alimentaire, environnementale et zoonotique, Agence de la santé \\ publique du Canada, Guelph (Ontario) \\ ${ }^{*}$ Correspondance à : rita.finley@phac-aspc.gc.ca
}

\section{Résumé d'article}

Objectif : Décrire les profils de recommandations d'antibiotiques par les médecins de cabinets médicaux de cinq régions au Canada entre 2007 et 2011.

Méthodologie : Les valeurs fournies sont des estimations fondées sur les données trimestrielles recueillies auprès d'un échantillon d'environ 652 médecins stratifié par région et spécialité. Durant quatre trimestres consécutifs, chaque médecin a tenu à jour un journal dans lequel il consignait des renseignements détaillés sur toutes les consultations de patients menées durant une période de 48 heures choisie au hasard. Cette information a été extrapolée par la suite au moyen d'un facteur de projection afin d'estimer les ordonnances rédigées par tous les médecins du Canada.

Résultats : Durant la période de surveillance de cinq ans, les médecins ont établi près de 1,5 milliard de diagnostics et fourni un total d'environ 120 millions de recommandations d'antimicrobiens. En 2011 seulement, 289 millions de diagnostics cliniques ont été posés, parmi lesquels $8 \%$ ont entraîné la recommandation d'un antimicrobien. La majorité de ces antimicrobiens $(51 \%)$ visaient le traitement de maladies de l'appareil respiratoire, $14 \%$ le traitement d'infections des voies urinaires et $11 \%$ le traitement de maladies de la peau et des tissus sous-cutanés. Les recommandations d'antimicrobiens étaient les plus nombreuses chez les patients des groupes d'âge 0 à 2 ans, 3 à 9 ans et 65 ans ou plus. Les taux de recommandations d'antimicrobiens ont diminué de façon générale entre les années 2007 et 2011, sauf pour les maladies de l'appareil génito-urinaire et les maladies de l'oreille, lesquels sont demeurés stables. Globalement, les antimicrobiens les plus souvent recommandés comprenaient les macrolides, les pénicillines à large spectre et les fluoroquinolones. Il est à noter que le nombre de recommandations de céphalosporines de troisième génération, bien que moins élevé, a augmenté de $42 \%$.

Conclusion : À l'exception des céphalosporines de troisième génération, le pourcentage de recommandations d'antimicrobiens par les médecins de cabinets médicaux au Canada est demeuré stable ou a diminué entre 2007 et 2011. Le nombre et le taux de recommandations d'antimicrobiens différaient entre les provinces, la région de l'Atlantique et le Québec affichant des taux plus élevés de recommandations d'antimicrobiens comparativement à l'ensemble du pays.

\section{Introduction}

Le Programme intégré canadien de surveillance de la résistance aux antimicrobiens (PICRA) surveille les tendances en matière de résistance aux antimicrobiens et d'utilisation d'antimicrobiens chez certains organismes bactériens isolés de sources humaines, animales et alimentaires de partout au Canada. Le programme est fondé sur plusieurs composantes de surveillance représentatives et méthodologiquement unifiées qui peuvent être liées afin d'examiner la relation entre les antimicrobiens utilisés chez les animaux destinés à la consommation et les humains, ainsi que les répercussions subséquentes sur la santé. Ces renseignements permettent (i) la création de politiques basées sur des données probantes afin de contrôler l'utilisation des antimicrobiens dans les hôpitaux, au sein de la communauté et dans les 
milieux agricoles, permettant ainsi de prolonger l'efficacité de ces médicaments, ainsi que (ii) l'identification de mesures appropriées pour freiner l'apparition de bactéries résistantes et leur propagation entre les animaux, les aliments et les humains au Canada.

Les rapports de surveillance de l'utilisation des antimicrobiens chez les humains sont publiés sur une base régulière, et ce, afin de fournir un aperçu national annuel des pratiques actuelles en matière d'utilisation des antimicrobiens au Canada, d'établir des comparaisons, et finalement, de favoriser la gestion des antibiotiques et leur inscription aux listes de médicaments. L'objectif de cet article est de résumer le rapport le plus récent du PICRA qui décrit les profils de recommandations d'antibiotiques par les médecins de cabinets médicaux de cinq régions au Canada entre 2007 et 2011 (1).

\section{Méthodologie}

L'ensemble de données de l'Index canadien des maladies et traitements est acheté par l'Agence de la santé publique du Canada auprès d'IMS Health Canada Inc. Cet ensemble de données fournit de l'information sur les formes de maladies rencontrées et les types de traitements prescrits par les médecins de cabinets médicaux de cinq régions du Canada : Atlantique (Nouveau-Brunswick, TerreNeuve-et-Labrador, Nouvelle-Écosse et Île-du-Prince-Édouard), Québec, Ontario, provinces des Prairies (Alberta, Manitoba et Saskatchewan) et Colombie-Britannique). Les valeurs fournies sont des estimations fondées sur les données trimestrielles recueillies auprès d'un échantillon d'environ 652 médecins stratifié par région et spécialité. Durant quatre trimestres consécutifs, chaque médecin tient à jour un journal dans lequel il consigne des renseignements détaillés sur toutes les consultations de patients menées durant une période de 48 heures choisie au hasard. Cette information est ensuite extrapolée au moyen d'un facteur de projection afin d'estimer l'« univers » constitué d'environ 52959 médecins, sensiblement représentatif de l'ensemble des données canadiennes.

L'information contenue dans cette analyse concerne les antimicrobiens pour lesquels un médecin a émis une recommandation ou une ordonnance, et ne représente pas les ordonnances effectivement exécutées par les pharmaciens ni consommées par les patients. Ces données n'incluent pas les consultations d'infirmiers de premier recours. D'autre part, les visites de diagnostic ne correspondent pas au nombre total de patients, puisque certains patients font de multiples visites pour les mêmes raisons, tandis que d'autres patients peuvent recevoir plusieurs diagnostics. Les méthodes de collecte des données font en sorte que la taille de l'échantillon est parfois considérée comme trop petite pour être utile à la prise de décision. Toutefois, ces renseignements sont tout de même inclus dans cette analyse afin de fournir un aperçu des pratiques qui pourraient nécessiter des études ultérieures.

\section{Résultats}

Durant la période de surveillance de cinq ans (2007 à 2011), les médecins ont établi près de 1,5 milliard de diagnostics et fournit un total d'environ 121 millions de recommandations d'antimicrobiens (1). En 2011, un total de 289 millions de diagnostics cliniques ont été posés, parmi lesquels $8 \%$ ont entraîné la recommandation d'un antimicrobien (Tableau 1). Au cours de cette année, parmi tous les antimicrobiens recommandés par les médecins de cabinets médicaux, 51,3\% concernaient le traitement de maladies de l'appareil respiratoire, le traitement d'infections des voies urinaires $(14,5 \%)$ et le traitement des maladies de la peau et des tissus sous-cutanés (11,3\%). 
TABLEAU 1. Nombre total de diagnostics posés en cabinet, taux de diagnostics, nombre total de recommandations d'antimicrobiens, taux de recommandations d'antimicrobiens et pourcentage de diagnostics entraînant la recommandation d'un antimicrobien par les médecins de cabinets médicaux au Canada, par classe de diagnostic, en 2011

\begin{tabular}{|c|c|c|c|c|c|}
\hline Classe de diagnostic & $\begin{array}{c}\text { Nombre total } \\
\text { de } \\
\text { diagnostics }\end{array}$ & $\mid \begin{array}{c}\text { Total de } \\
\text { diagnostics / } \\
10000 \text { habitant } \\
\text { s }\end{array}$ & $\mid \begin{array}{c}\text { Recommandati } \\
\text { ons } \\
\text { d'antimicrobien } \\
\mathrm{S}(\mathrm{N})\end{array}$ & \begin{tabular}{|l|} 
Nombre total de \\
recommandations \\
d'antimicrobiens / \\
10000 habitants
\end{tabular} & $\begin{array}{c}\text { Pourcentage des } \\
\text { diagnostics ayant } \\
\text { donné lieu à la } \\
\text { recommandation } \\
\text { d'antimicrobiens }\end{array}$ \\
\hline Complications de la grossesse, de l'accouchement et puerpérales & 1044850 & 302 & 17180 & 5 & 1,6 \\
\hline Anomalies congénitales & 925330 & 267 & 21290 & 6 & 2,3 \\
\hline Maladies du sang et des organes hématopoḯtiques & 2593490 & 749 & 45160 & 13 & 1,7 \\
\hline Maladies de la peau et du tissu sous-cutané & 21784200 & 6295 & 2551830 & 737 & 11,7 \\
\hline Maladies du système nerveux central & 10591580 & 3061 & 148900 & 43 & 1,4 \\
\hline Maladies de l'appareil circulatoire & $\underline{33884750}$ & $\underline{9791}$ & $\underline{62510}$ & $\underline{18}$ & $\underline{0,2}$ \\
\hline Maladies de l'oreille & $\underline{7627370}$ & $\underline{2204}$ & $\underline{2232080}$ & $\underline{645}$ & $\underline{29,3}$ \\
\hline Maladies de l'appareil digestif & 1674160 & 484 & 107900 & 31 & 6,4 \\
\hline Maladies du système génito-urinaire & 12878470 & 3721 & 821180 & 237 & 6,4 \\
\hline Maladies de l'appareil respiratoire & 37079860 & 10715 & 11628520 & 3360 & 31,4 \\
\hline $\begin{array}{l}\text { Maladies endocriniennes, nutritionnelles et métaboliques et troubles } \\
\text { immunitaires }\end{array}$ & 28537420 & 8246 & 62310 & 18 & 0,2 \\
\hline Infections des voies urinaires & 6951220 & 2009 & 3285390 & 949 & 47,3 \\
\hline Lésions traumatiques et empoisonnements & 11575360 & 3345 & 317020 & 92 & 2,7 \\
\hline Maladies musculosquelettiques & 28086920 & 8116 & 136030 & 39 & 0,5 \\
\hline Tumeurs & 7124130 & 2059 & 93740 & 27 & 1,3 \\
\hline Autres maladies de l'appareil digestif & 17401060 & 5028 & 684730 & 198 & 3,9 \\
\hline Affections périnatales & 438380 & 127 & 14540 & 4 & 3,3 \\
\hline Classifications supplémentaires & 37076290 & 10714 & 77420 & 22 & 0,2 \\
\hline Symptômes et états morbides mal définis & 21771660 & 6291 & 359500 & 104 & 1,7 \\
\hline Total & 289046500 & 83524 & 22667230 & 6550 & 7,8 \\
\hline
\end{tabular}

Les antimicrobiens étaient recommandés dans tous les groupes d'âge en 2011, les taux étant proportionnellement plus élevés chez les patients du groupe d'âge des 0 à 2 ans (12 recommandations d'antimicrobiens par 10 habitants), suivis des 3 à 9 ans (9 recommandations d'antimicrobiens par 10 habitants), puis des 60 à 64 ans et des 65 ans et plus (7 recommandations d'antimicrobiens par 10 habitants dans chacun de ces groupes d'âge) (Tableau 2). 
TABLEAU 2. Taux de diagnostics, taux de recommandations d'antimicrobiens et pourcentage de diagnostics avec recommandation d'un antimicrobien par les médecins de cabinets médicaux au Canada, par groupe d'âge et par sexe, 2011

\begin{tabular}{|c|c|c|c|}
\hline $\begin{array}{l}\text { Caractéristiques } \\
\text { démographiques : } \\
\text { âge (année) ou sexe }\end{array}$ & $\begin{array}{l}\text { Nombre de } \\
\text { diagnostics / } \\
10 \text { habitants }^{1}\end{array}$ & $\begin{array}{c}\text { Recom mandations } \\
\text { d'antimicrobiens / } \\
10 \text { habitants }^{2}\end{array}$ & $\begin{array}{c}\text { Pourcentage des } \\
\text { diagnostics ayant donné } \\
\text { lieu à la recommandation } \\
\text { d'antimicrobiens }\end{array}$ \\
\hline \multicolumn{4}{|l|}{ Âge } \\
\hline 0 à 2 & 123 & 12 & 9,7 \\
\hline 3 à 9 & 52 & 9 & 16,5 \\
\hline 10 à 19 & 42 & 6 & 13,0 \\
\hline 20 à 39 & 59 & 6 & 10,7 \\
\hline 40 à 59 & 81 & 5 & 6,6 \\
\hline 60 à 64 & 122 & 7 & 5,5 \\
\hline 65 et plus & 152 & 7 & 4,9 \\
\hline \multicolumn{4}{|l|}{ Sexe } \\
\hline Femmes & 93 & 7 & 7,7 \\
\hline Hommes & 71 & 6 & 8,0 \\
\hline
\end{tabular}

${ }^{1}$ Le nombre de diagnostics ne représente pas le nombre de consultations pour une personne donnée, mais le nombre de diagnostics posés; si une personne présente plusieurs pathologies, chaque diagnostic (pathologie) est consigné(e) séparément.

${ }^{2}$ Les données de ce tableau ne représentent pas les ordonnances effectivement exécutées par les pharmaciens ni les produits consommés par les patients, car aucune information sur l'observance des traitements par les patients n'était disponible.

Pour la plupart des classes d'antimicrobiens, le pourcentage de recommandations par les médecins de cabinets médicaux au Canada est demeuré stable ou a diminué entre 2007 et 2011 (Figure 1). De légères augmentations ont été observées en présence de complications puerpérales, de grossesse ou d'accouchement; d'anomalies congénitales; de maladies de l'appareil digestif; de blessures et empoisonnements; et d'affections périnatales. 
FIGURE 1. Pourcentage de diagnostics ayant donné lieu à la recommandation d'un traitement antimicrobien par les médecins de cabinets médicaux au Canada, en 2007 et de 2010 à 2011

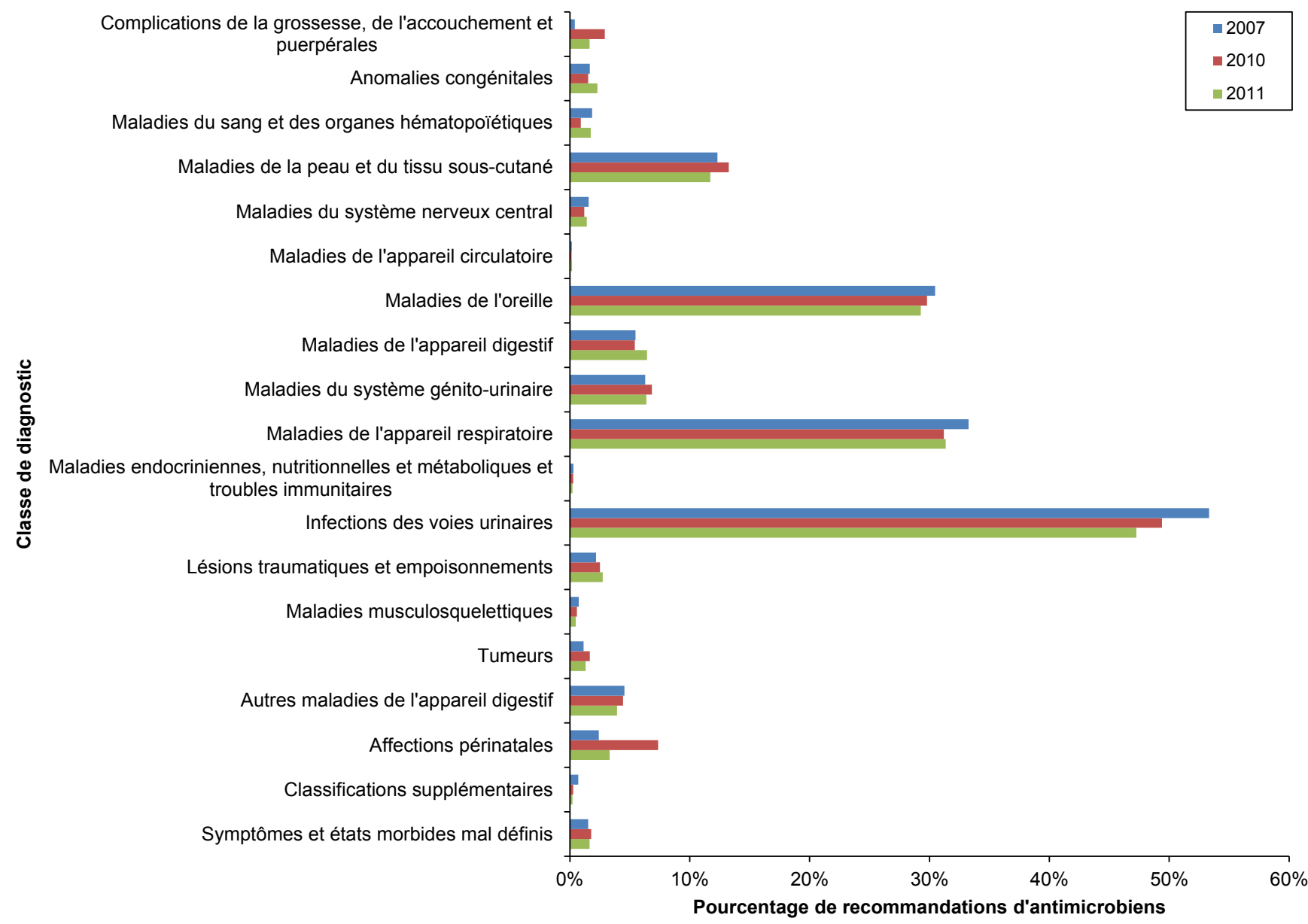

Les antimicrobiens les plus souvent recommandés en 2011 étaient les macrolides

(1 638 recommandations par 10000 habitants), les pénicillines à large spectre (1504 recommandations par 10000 habitants) et les fluoroquinolones (1 202 recommandations par 10000 habitants). Toutefois, des augmentations importantes du nombre de recommandations de céphalosporines de troisième génération ont été observées (augmentation de $42 \%$ comparativement à 2007), lesquelles étaient attribuables à une augmentation des recommandations pour le traitement de maladies de l'appareil respiratoire, le traitement de maladies de l'appareil génito-urinaire et le traitement d'infections des voies urinaires.

Des variations interprovinciales ont été observées au niveau du nombre total de recommandations d'antimicrobiens par 10000 habitants, la région de l'Atlantique (7,101 recommandations par 10000 habitants) et le Québec (8,268 recommandations par 10000 habitants) affichant les taux les plus élevés comparativement à l'ensemble du pays. Les taux de diagnostics et de recommandations d'antimicrobiens par 10000 habitants variaient selon les provinces et les maladies à traiter. Différentes préférences quant à l'antimicrobien de choix pour le traitement des maladies étaient également observées entre les provinces (Tableau 3). 
TABLEAU 3. Taux de diagnostics, taux de recommandations d'antimicrobiens et pourcentage de diagnostics avec recommandation d'un antimicrobien par les médecins de cabinets médicaux au Canada, par classe de diagnostic et par province/région, 2011

\begin{tabular}{|c|c|c|c|c|}
\hline Classe de diagnostic & Région & $\begin{array}{c}\text { Nombre de } \\
\text { diagnostics / } \\
10000 \text { habitants }^{1}\end{array}$ & $\begin{array}{l}\text { Nom bre de } \\
\text { recommandations } \\
\text { d'antim icrobiens / } \\
10000 \text { habitants }^{2}\end{array}$ & $\begin{array}{l}\text { Pourcentage des } \\
\text { diagnostics ayant } \\
\text { donné lieu à la } \\
\text { recommandation } \\
\text { d'antimicrobiens }\end{array}$ \\
\hline \multirow{6}{*}{$\begin{array}{l}\text { Complications de la grossesse, de } \\
\text { l'accouchement et puerpérales }\end{array}$} & Colombie-Britannique & 350 & $\mathrm{AAP}^{3}$ & AAP \\
\hline & Prairies $^{4}$ & 303 & 3 & 1,1 \\
\hline & Ontario & 271 & AAP & AAP \\
\hline & Québec & 330 & 19 & 5,7 \\
\hline & Atlantique $^{5}$ & 298 & AAP & AAP \\
\hline & Total & 302 & 5 & 1,6 \\
\hline \multirow{6}{*}{ Anomalies congénitales } & Colombie-Britannique & 374 & 10 & 2,7 \\
\hline & Prairies & 265 & 13 & 4,7 \\
\hline & Ontario & 171 & 5 & 2,9 \\
\hline & Québec & 296 & AAP & AAP \\
\hline & Atlantique & 530 & 10 & 1,9 \\
\hline & Total & 267 & 6 & 2,3 \\
\hline \multirow{6}{*}{$\begin{array}{l}\text { Maladies du sang et des organes } \\
\text { hématopoiétiques }\end{array}$} & Colombie-Britannique & 715 & AAP & AAP \\
\hline & Prairies & 665 & 12 & 1,8 \\
\hline & Ontario & 744 & 20 & 2,7 \\
\hline & Québec & 707 & 13 & 1,9 \\
\hline & Atlantique & 1242 & AAP & AAP \\
\hline & Total & 749 & 13 & 1,7 \\
\hline \multirow{6}{*}{$\begin{array}{l}\text { Maladies de la peau et du tissu sous- } \\
\text { cutané }\end{array}$} & Colombie-Britannique & 7602 & 820 & 10,8 \\
\hline & Prairies & 6061 & 706 & 11,6 \\
\hline & Ontario & 5866 & 792 & 13,5 \\
\hline & Québec & 6399 & 664 & 10,4 \\
\hline & Atlantique & 6741 & 631 & 9,4 \\
\hline & Total & 6295 & 737 & 11,7 \\
\hline \multirow{6}{*}{$\begin{array}{l}\text { Maladies du système nerveux } \\
\text { central }\end{array}$} & Colombie-Britannique & 3472 & 73 & 2,1 \\
\hline & Prairies & 2440 & 33 & 1,3 \\
\hline & Ontario & 3297 & 39 & 1,2 \\
\hline & Québec & 3020 & 37 & 1,2 \\
\hline & Atlantique & 2811 & 57 & 2,0 \\
\hline & Total & 3061 & 43 & 1,4 \\
\hline \multirow{6}{*}{ Maladies de l'appareil circulatoire } & Colombie-Britannique & 9503 & 48 & 0,5 \\
\hline & Prairies & 9231 & 5 & 0,0 \\
\hline & Ontario & 9479 & 19 & 0,2 \\
\hline & Québec & 1037 & 8 & 0,8 \\
\hline & Atlantique & 12081 & 24 & 0,2 \\
\hline & Total & 9792 & 18 & 0,2 \\
\hline
\end{tabular}

${ }^{1}$ Le nombre de diagnostics ne représente pas le nombre de consultations pour une personne donnée, mais le nombre de diagnostics posés; si une personne vient consulter pour plusieurs pathologies, chaque diagnostic (pathologie) est consigné(e) séparément.

${ }^{2}$ Les données de ce tableau ne représentent pas les ordonnances effectivement exécutées par les pharmaciens ni les produits consommés par les patients, car aucune information sur l'observance des traitements par les patients n'était disponible.

${ }^{3} \mathrm{AAP}=$ Aucun antimicrobien proposé (recommandé).

${ }^{4}$ Les Prairies regroupent les provinces de l'Alberta, de la Saskatchewan et du Manitoba.

${ }^{5}$ La région de l'Atlantique comprend les provinces du Nouveau-Brunswick, de la Nouvelle-Écosse, de l'île-du-Prince-Édouard et de Terre-Neuve-et-Labrador. 
TABLEAU 3 (suite). Taux de diagnostics, taux de recommandations d'antimicrobiens et pourcentage de diagnostics avec recommandation d'un antimicrobien par les médecins de cabinets médicaux au Canada, par classe de diagnostic et par province/région, 2011

\begin{tabular}{|c|c|c|c|c|}
\hline Classe de diagnostic & Région & $\begin{array}{c}\text { Nombre de } \\
\text { diagnostics / } \\
10000 \text { habitants }^{1}\end{array}$ & $\begin{array}{l}\text { Nom bre de } \\
\text { recommandations } \\
\text { d'antim icrobiens / } 10000 \text { habitants }^{2}\end{array}$ & $\begin{array}{l}\text { Pourcentage des } \\
\text { diagnostics ayant } \\
\text { donné lieu à la } \\
\text { recommandation } \\
\text { d'antimicrobiens }\end{array}$ \\
\hline \multirow{6}{*}{ Maladies de l'oreille } & Colombie-Britannique & 2285 & 349 & 15,3 \\
\hline & Prairies $^{4}$ & 1628 & 460 & 28,2 \\
\hline & Ontario & 1708 & 514 & 30,1 \\
\hline & Québec & 3123 & 1168 & 37,4 \\
\hline & Atlantique $^{5}$ & 3348 & 702 & 21,0 \\
\hline & Total & 2204 & 645 & 29,3 \\
\hline \multirow{6}{*}{ Maladies de l'appareil digestif } & Colombie-Britannique & 452 & 53 & 11,6 \\
\hline & Prairies & 521 & 9 & 1,7 \\
\hline & Ontario & 461 & 29 & 6,4 \\
\hline & Québec & 530 & 28 & 5,3 \\
\hline & Atlantique & 445 & 69 & 15,6 \\
\hline & Total & 484 & 31 & 6,4 \\
\hline \multirow{6}{*}{ Maladies du système génito-urinaire } & Colombie-Britannique & 3646 & 207 & 5,7 \\
\hline & Prairies & 3500 & 252 & 7,2 \\
\hline & Ontario & 3606 & 218 & 6,0 \\
\hline & Québec & 3815 & 277 & 7,3 \\
\hline & Atlantique & 4957 & 243 & 4,9 \\
\hline & Total & 3722 & 237 & 6,4 \\
\hline \multirow{6}{*}{ Maladies de l'appareil respiratoire } & Colombie-Britannique & 9699 & 2649 & 27,3 \\
\hline & Prairies & 10193 & 3307 & 32,4 \\
\hline & Ontario & 9687 & 3000 & 31,0 \\
\hline & Québec & 13044 & 4377 & 33,6 \\
\hline & Atlantique & 12504 & 3641 & 29,1 \\
\hline & Total & 10715 & 3360 & 31,4 \\
\hline \multirow{6}{*}{$\begin{array}{l}\text { Maladies endocriniennes, } \\
\text { nutritionnelles et métaboliques et } \\
\text { troubles immunitaires }\end{array}$} & Colombie-Britannique & 7353 & $\mathrm{AAP}^{3}$ & AAP \\
\hline & Prairies & 6940 & 14 & 0,2 \\
\hline & Ontario & 8557 & 23 & 0,3 \\
\hline & Québec & 8477 & 28 & 0,3 \\
\hline & Atlantique & 11215 & 5 & $<0,1$ \\
\hline & Total & 8247 & 18 & 0,2 \\
\hline \multirow{6}{*}{ Infections des voies urinaires } & Colombie-Britannique & 2241 & 982 & 43,8 \\
\hline & Prairies & 1886 & 825 & 43,8 \\
\hline & Ontario & 1869 & 855 & 45,7 \\
\hline & Québec & 1891 & 1143 & 60,5 \\
\hline & Atlantique & 3164 & 1134 & 35,8 \\
\hline & Total & 2009 & 949 & 47,3 \\
\hline
\end{tabular}

${ }^{1}$ Le nombre de diagnostics ne représente pas le nombre de consultations pour une personne donnée, mais le nombre de diagnostics posés; si une personne vient consulter pour plusieurs pathologies, chaque diagnostic (pathologie) est consigné(e) séparément.

${ }^{2}$ Les données de ce tableau ne représentent pas les ordonnances effectivement exécutées par les pharmaciens ni les produits consommés par les patients, car aucune information sur l'observance des traitements par les patients n'était disponible.

${ }^{3} \mathrm{AAP}=$ Aucun antimicrobien proposé (recommandé).

${ }^{4}$ Les Prairies regroupent les provinces de l'Alberta, de la Saskatchewan et du Manitoba.

${ }^{5}$ La région de l'Atlantique comprend les provinces du Nouveau-Brunswick, de la Nouvelle-Écosse, de l'île-du-Prince-Édouard et de Terre-Neuve-et-Labrador. 
TABLEAU 3 (suite). Taux de diagnostics, taux de recommandations d'antimicrobiens et pourcentage de diagnostics avec recommandation d'un antimicrobien par les médecins de cabinets médicaux au Canada, par classe de diagnostic et par province/région, 2011

\begin{tabular}{|c|c|c|c|c|}
\hline Classe de diagnostic & Région & $\begin{array}{c}\text { Nombre de } \\
\text { diagnostics / } \\
10000 \text { habitants }^{1}\end{array}$ & $\begin{array}{l}\text { Nombre de } \\
\text { recom mandations } \\
\text { d'antimicrobiens / } \\
10000 \text { habitants }^{2}\end{array}$ & $\begin{array}{l}\text { Pourcentage des } \\
\text { diagnostics ayant } \\
\text { donné lieu à la } \\
\text { recommandation } \\
\text { d'antimicrobiens }\end{array}$ \\
\hline \multirow{6}{*}{$\begin{array}{l}\text { Lésions traumatiques et } \\
\text { empoisonnements }\end{array}$} & Colombie-Britannique & 5400 & 99 & 1,8 \\
\hline & Prairies $^{4}$ & 3446 & 93 & 2,7 \\
\hline & Ontario & 2864 & 94 & 3,3 \\
\hline & Québec & 3221 & 90 & 2,8 \\
\hline & Atlantique $^{5}$ & 2398 & 73 & 3,0 \\
\hline & Total & 3345 & 92 & 2,7 \\
\hline \multirow{6}{*}{ Maladies musculosquelettiques } & Colombie-Britannique & 8402 & 4 & $<0,1$ \\
\hline & Prairies & 8748 & 53 & 0,6 \\
\hline & Ontario & 7911 & 56 & 0,7 \\
\hline & Québec & 6804 & 13 & 0,2 \\
\hline & Atlantique & 11928 & 69 & 0,6 \\
\hline & Total & 8116 & 39 & 0,5 \\
\hline \multirow{6}{*}{ Tumeurs } & Colombie-Britannique & 2012 & 10 & 0,5 \\
\hline & Prairies & 1630 & 3 & 0,2 \\
\hline & Ontario & 1840 & 8 & 0,5 \\
\hline & Québec & 2645 & 95 & 3,6 \\
\hline & Atlantique & 2614 & $\mathrm{AAP}^{3}$ & AAP \\
\hline & Total & 2059 & 27 & 1,3 \\
\hline \multirow{6}{*}{ Autres maladies de l'appareil digestif } & Colombie-Britannique & 4925 & 237 & 4,8 \\
\hline & Prairies & 5460 & 138 & 2,5 \\
\hline & Ontario & 4547 & 214 & 4,7 \\
\hline & Québec & 4962 & 224 & 4,5 \\
\hline & Atlantique & 7311 & 106 & 1,5 \\
\hline & Total & 5028 & 198 & 3,9 \\
\hline \multirow{6}{*}{ Affections périnatales } & Colombie-Britannique & 123 & AAP & AAP \\
\hline & Prairies & 154 & 16 & 10,1 \\
\hline & Ontario & 151 & AAP & AAP \\
\hline & Québec & 96 & 6 & 6,5 \\
\hline & Atlantique & 35 & AAP & AAP \\
\hline & Total & 127 & 4 & 3,3 \\
\hline \multirow{6}{*}{ Classifications supplémentaires } & Colombie-Britannique & 10174 & 12 & 0,1 \\
\hline & Prairies & 12776 & 9 & 0,1 \\
\hline & Ontario & 10851 & 24 & 0,2 \\
\hline & Québec & 9323 & 37 & 0,4 \\
\hline & Atlantique & 10867 & 21 & 0,2 \\
\hline & Total & 10714 & 22 & 0,2 \\
\hline \multirow{6}{*}{$\begin{array}{l}\text { Symptômes et états morbides mal } \\
\text { définis }\end{array}$} & Colombie-Britannique & 6427 & 97 & 1,5 \\
\hline & Prairies & 5819 & 68 & 1,2 \\
\hline & Ontario & 6666 & 123 & 1,9 \\
\hline & Québec & 4792 & 41 & 0,9 \\
\hline & Atlantique & 10502 & 315 & 3,0 \\
\hline & Total & 6291 & 104 & 1,7 \\
\hline
\end{tabular}


${ }^{1}$ Le nombre de diagnostics ne représente pas le nombre de consultations pour une personne donnée, mais le nombre de diagnostics posés; si une personne vient consulter pour plusieurs pathologies, chaque diagnostic (pathologie) est consigné(e) séparément.

${ }^{2}$ Les données de ce tableau ne représentent pas les ordonnances effectivement exécutées par les pharmaciens ni les produits consommés par les patients, car aucune information sur l'observance des traitements par les patients n'était disponible.

${ }^{3} \mathrm{AAP}=$ Aucun antimicrobien proposé (recommandé).

${ }^{4}$ Les Prairies regroupent les provinces de l'Alberta, de la Saskatchewan et du Manitoba.

${ }^{5}$ La région de l'Atlantique comprend les provinces du Nouveau-Brunswick, de la Nouvelle-Écosse, de l'île-du-Prince-Édouard et de Terre-Neuve-et-Labrador.

\section{Discussion}

Huit pour cent ( $8 \%)$ des diagnostics cliniques posés par les médecins de cabinets médicaux ont entraîné la recommandation d'un antimicrobien en 2011. Cinquante et un pour cent (51\%) de ces antimicrobiens visaient le traitement de maladies de l'appareil respiratoire, $14 \%$ le traitement d'infections des voies urinaires et $11 \%$ le traitement de maladies de la peau et des tissus sous-cutanés. Les recommandations d'antimicrobiens étaient les plus nombreuses chez les patients des groupes d'âge 0 à 2 ans, 3 à 9 ans et 65 ans ou plus. Les taux de recommandations d'antimicrobiens ont diminué entre les années 2007 et 2011 pour les maladies de l'oreille, de l'appareil respiratoire, les infections des voies urinaires et les maladies de la peau et des tissus sous-cutanés, alors que les taux associés aux maladies de l'appareil génito-urinaire sont demeurés stables.

Globalement, les antimicrobiens les plus souvent recommandés comprenaient les macrolides, les pénicillines à large spectre et les fluoroquinolones. Des différences interprovinciales étaient observées au niveau des taux de recommandations d'antimicrobiens et aussi des antimicrobiens sélectionnés comme traitement, la région de l'Atlantique et le Québec affichant des taux plus élevés de recommandations d'antimicrobiens comparativement à l'échelle nationale. La Colombie-Britannique détenait le taux le plus faible de recommandations d'antimicrobiens pour les maladies de l'appareil respiratoire, alors que le Québec et la région de l'Atlantique détenaient les taux les plus élevés pour les maladies de l'oreille. Les Prairies détenaient le taux le plus faible de recommandations d'antimicrobiens pour les infections des voies urinaires (à noter toutefois qu'une augmentation de ce taux a été observée entre 2010 et 2011). Un taux similaire de recommandations d'antimicrobiens a été observé dans tout le pays pour ce qui est du traitement des maladies de l'appareil génito-urinaire, alors que des diminutions des recommandations étaient observées dans tout le pays pour les maladies de la peau et des tissus sous-cutanés.

L'ensemble de données de l'Index canadien des maladies et traitements montre plusieurs limites et restrictions. Les médicaments indiqués sont ceux prescrits ou recommandés par les médecins et ne représentent pas les ordonnances effectivement exécutées par les pharmaciens ni les produits consommés par les patients, puisque l'information sur l'observance des traitements n'était pas disponible. Ces données n'incluent pas les consultations d'infirmiers de premier recours. D'autre part, les visites de diagnostic ne correspondent pas au nombre total de patients, puisque certains patients peuvent faire plusieurs visites pour les mêmes raisons, tandis que d'autres patients peuvent recevoir plusieurs diagnostics.

Les médicaments indiqués peuvent comprendre des échantillons qui ne figurent pas nécessairement sur une ordonnance exécutée puisque les médecins consignent tous les traitements suggérés. Les médecins consignent également les « médicaments antérieurement prescrits dont la prise est à continuer » pour un diagnostic donné, ce qui ne donne pas nécessairement lieu à l'exécution d'une ordonnance. En outre, certains traitements médicamenteux et certains diagnostics sont sous-représentés en raison de l'automédication (p. ex., achat de produits en vente libre). Finalement, certains échantillons, jugés trop petits pour être utiles à la prise de décision, ont toutefois été inclus à titre informatif uniquement; comme les erreurs d'échantillonnage peuvent nuire à la fiabilité des données, la prudence s'impose lors de l'interprétation des données relatives à des catégories de maladies reposant sur un échantillon de petite taille. Les données ne sont disponibles qu'à l'échelle des régions, où les fluctuations sont plus ou moins évidentes et aucune information spécifique sur les provinces individuelles n'a pu être déterminée. 
La résistance aux antimicrobiens continuera à mettre en danger la santé des Canadiens et des autres habitants de la planète pendant encore un certain temps. La surveillance de l'utilisation des antibiotiques aidera à documenter les progrès accomplis dans la réduction de cette résistance ainsi que les répercussions possibles de ces changements sur la résistance globale aux antibiotiques.

\section{Remerciements}

L'auteur aimerait souligner la contribution des réviseurs externes du Rapport sur l'utilisation des antimicrobiens chez les humains : $D^{r}$ John Conly, $D^{r}$ Jim Hutchinson, $D^{r}$ Warren Mclsaac, $D^{r}$ David Patrick, $D^{\text {re }}$ Lynora Saxinger, $D^{r}$ Daniel Thirion et $D^{r}$ Karl Weiss. Sans leur étroite collaboration et leur participation, l'analyse et la présentation de ces données n'auraient pas été possible.

\section{Conflit d'intérêts}

II n'y a aucun conflit d'intérêts à déclarer.

\section{Financement}

Ce travail a été appuyé par l'Agence de la santé publique du Canada.

\section{Avertissement}

Cet article a été préparé grâce aux données d'IMS Health Canada Inc. Les analyses, les conclusions, les opinions ainsi que les énoncés exprimés dans cet article sont ceux des auteurs et non ceux d'IMS Health Canada Inc.

\section{Références}

(1) Agence de la santé publique du Canada (ASPC). Programme intégré canadien de surveillance de la résistance aux antimicrobiens (PICRA) : Rapport sommaire sur la résistance aux antimicrobiens 2011. Guelph (Ont.) : ASPC; 2012. http://publications.gc.ca/collections/collection_2013/aspc-phac/HP2-4-2-2011-fra.pdf 